\title{
Program Information
}

- Therapy General Poster Discussion

- All Therapy General Poster Discussion

- All Sessions

- Program Home

\section{A Micro-Raman Spectroscopy Study of the Dose-Dependence of EBT3 GafChromic ${ }^{\mathrm{TM}}$ Films for Quantifying the Degree of Molecular Polymerization}

\begin{abstract}
M Callens ${ }^{1 *}$, W Crijns ${ }^{2}$, V Simons ${ }^{3}$, I De Wolf ${ }^{3,6}$, T Depuydt ${ }^{2}, \mathrm{~F} \mathrm{Maes}^{2}$, $\mathrm{K}$ Haustermans $^{2}$, $J^{\text {J'hooge }}{ }^{4}$, E D'Agostino ${ }^{5}$, H Pfeiffer ${ }^{6}$, K Van Den Abeele ${ }^{1}$, (1) Department of Physics, Wave Propagation and Signal Processing, KU Leuven KULAK, Kortrijk, Belgium, (2) Department of Radiation Oncology, KU Leuven, Leuven, Belgium, (3) IMEC, Leuven, Belgium, (4) Department of Cardiovascular Sciences, Bio-Medical Science Group, KU Leuven, Leuven, Belgium, (5) DoseVue NV, Hasselt, Belgium, (6) Department of Materials Engineering, KU Leuven, Leuven, Belgium
\end{abstract}

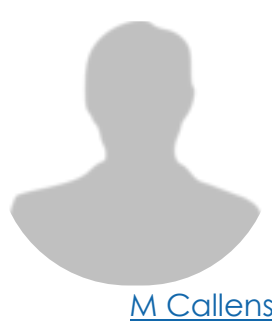

Presentations

SU-E-T-44 (Sunday, July 12, 2015) 3:00 PM - 6:00 PM Room: Exhibit Hall

Purpose: Radiochromic films, such as the poly-diacetylene-based EBT3 GafChromic ${ }^{\text {TM }}$ films (Ashland Specialty Ingredients, Wayne, NY, USA), are widely used for dosimetry applications because of their clear energy independence, high spatial resolution, near tissue equivalence and easy handling. The films undergo a slight color change by radiation-induced polymerization of diacetylene monomers. But more importantly, the film becomes optically less transparent with increasing radiation dose, with a saturation starting between 10 and 20 Gy, i.e. a common SBRT dose level. In contrast to the chromatic properties, less attention has been given to the underlying molecular mechanism that induces this partial color change and strongly reduces the transparency. Therefore, the current work investigates the variation of the molecular composition of the active layer of EBT3 films for an SBRT dose range.

Method: Uncoated EBT3 films were irradiated with a $6 \mathrm{MV}$ photon beam using dose levels between 0 and 20 Gy. The relative variation of the polymer content as a function of the applied radiation dose was studied using micro-Raman spectroscopy. Raman spectroscopy with a $633 \mathrm{~nm}$ probe laser incident on the active layer allowed to identify the film constituents and to estimate the amount of poly-diacetylenes from the intensities of the unique molecular vibrations of the molecule.

Results: The normalized intensity of all polymeric vibrations, and most notably the polymeric triple and double carbon-carbon bonds at $2058 \mathrm{~cm}^{-1}$ and $1446 \mathrm{~cm}^{-1}$ respectively, increase with increasing dose up to a saturation level starting at about $10 \mathrm{~Gy}$, indicating a corresponding increase and saturation of the amount of polymers. This molecular saturation process is the main cause of the non-linear dose response (i.e. a transparency reduction) and of the limited dose range of the studied films.

Conclusion: Raman spectroscopy provides new and more fundamental insights in the mechanism of the dosedependence of EBT3 films.

Funding Support, Disclosures, and Conflict of Interest: This work was funded by the Research Foundation Flanders (FWO).

Contact Email: maarten.callens@kuleuven.be 